\title{
Search Interfaces for Mathematicians
}

\author{
Andrea Kohlhase \\ Jacobs University Bremen and FIZ Karlsruhe
}

\begin{abstract}
Access to mathematical knowledge has changed dramatically in recent years, therefore changing mathematical search practices. Our aim with this study is to scrutinize professional mathematicians' search behavior. With this understanding we want to be able to reason why mathematicians use which tool for what search problem in what phase of the search process. To gain these insights we conducted 24 repertory grid interviews with mathematically inclined people (ranging from senior professional mathematicians to non-mathematicians). From the interview data we elicited patterns for the user group "mathematicians" that can be applied when understanding design issues or creating new designs for mathematical search interfaces.
\end{abstract}

\section{Introduction}

Mathematical practices are changing due to the availability of mathematical knowledge on the Web. This paper deals with the question whether mathematicians have special needs or preferences when accessing this knowledge and if yes, what are those? In particular, we focus on how mathematicians think of search on the Web: what are their cognitive categories, what kinds of searches do they distinguish, and which attributes do they associate with tools for math access?

The usability study [10 conducted interviews with mathematicians and essentially stated that mathematicians didn't know how to use the offerings of mathematical search interfaces. To get a better understanding we wanted to dig deeper. In 27 ZHAO concentrates on user-centric and math-aware requirements for math search. The former are based on mathematicians' specific information needs and search behaviors, the latter are the needs for structured indizes by the system. In contrast, we focus on eliciting attributions of existing math search interfaces by mathematicians versus non-mathematicians. We hope to learn what exactly sets mathematicians apart, since from this knowledge we can deduce implications for future mathematical designs.

We decided on using repertory grid interviews as main methodology to elicit evaluation schemes with respect to selected math search interfaces ("mSI") and to understand how mathematicians classify those mSIs. The main advantage of the method is its semi-empirical nature. On the one hand, it allows to get deep insights into the topic at hand through deconstruction and intense discussion of each subject's idiosyncratic set of constructs and their resp. mapping to the set of mSIs. On the other hand, the grids produced in such RGI sessions can be analyzed with a General Procrustes Analysis to obtain statistically significant 
correlations between the elicited constructs or the chosen mSIs. We used the Idiogrid 3 and the OpenRepGrid [21] software for this.

Information search is not a single act, but a process with many strategies and options: "In fact, we move fluidly between models of ask, browse, filter, and search without noting the shift. We scan feeds, ask questions, browse answers, and search again." 19, p.7]. Therefore, we can consider the term "search" as an umbrella term for (at least) the following approaches:

Finding = already knowing what one is looking for (20, 23] call it "factfinding")

Browsing $=$ getting an overview over a topic or an idea of a concept $(20]$ calls it "exploration of availability")

Surfing = surrendering to the links, drifting from one to another (see 26])

Solving/Information Gathering = creating a search plan, i.e., specifying a sequence of actions that achieves the solution of a problem (see [22, 65ff.], 88) Asking $=$ posing a question to find an answer (see [25])

Our question here is, what search approach is used with which assessment attributes for what kind of math search tool? The answer could enable us to design specifically for more math search approaches by learning from the used ones.

We start out in Section 2 with a description of the RGI study. In Section 3 we present the elicited interview data and note the patterns that emerge from this data. The patterns state interesting, prototypical attributions of mathematicians, which separate the data gathered from the group of mathematicians from the one of non-mathematicians. To demo the utility of such patterns, we apply them in a discussion of an interesting, confusing evaluation of two specific mSIs in Section 4. We conclude in Section 5 by hinting at general design implications for mathematical (search) interfaces based on the found set of patterns.

\section{The Study}

The aim of our study was to find out what distinguishes mathematicians from non-mathematicians when using a web interface for searching relevant content, here math content. From the outset it was clear that observational methods wouldn't work as the working context of a mathematician is typically neither restrained to certain locations nor time slots. Surveys (or structured interviews) were out of question as the answers require a deep insight of subjects into their own math search behavior, which cannot be assumed in general. Unstructured interviews could have been made use of to get such deep insights, but we would either have to do too many to be able to soundly interpret them or too few to draw general conclusions. Finally, the option of semi-structured interviews as methodology was discarded, since it became clear in the first pilot study trials that mathematicians tend to describe "truths" and "falsities". In particular, they try to scrutinize the interview or interviewer and manipulate the outcome towards what they think is the correct answer. Thus, the interviewer has to trade her observational stance with a continuously sparring stance, which hinders the process of gaining deep insights. 
In the end, we opted for the methodology of repertory grid interviews, as they allow a semi-empirical analysis, and interviewees understand quickly that they are not asked to decide on rights or wrongs. The Repertory Grid Interview (RGI) Technique 4, 7, 9] explores personal constructs, i.e., how persons perceive and understand the world around them. It has been used as a usability/user experience method to research users' personal constructs when interacting with software artifacts (see [5, 6, 24] for examples). RGI has the advantage that it can deliver valuable insights into the perception of users even with relative low numbers of study subjects (seeo 12 for more details).

\begin{tabular}{|c|c|c|}
\hline $\begin{array}{l}\text { Element } \\
\text { Name }\end{array}$ & Short Description & URL \\
\hline zbMathNew & $\begin{array}{l}\text { "an abstracting and reviewing service in pure and } \\
\text { applied mathematics" }\end{array}$ & zbMath.org \\
\hline zbMath0ld & the former interface of $\mathrm{zbMathNew}$ & not available \\
\hline MathSciNet & $\begin{array}{l}\text { "searchable database of reviews, abstracts and bib- } \\
\text { liographic information for much of the mathemat- } \\
\text { ical sciences literature" }\end{array}$ & ams.org/mathscinet \\
\hline $\begin{array}{l}\text { Google-Scho- } \\
\text { lar }\end{array}$ & $\begin{array}{l}\text { "search of scholarly literature across many disci- } \\
\text { plines and sources" }\end{array}$ & scholar.google.com \\
\hline Google & $\begin{array}{l}\text { "Search the world's information, including web- } \\
\text { pages, images, videos and more" }\end{array}$ & google.com \\
\hline myOffice & the personal office as math search interface & - \\
\hline TIB & The online catalogue of the Uni Hannover Library & tib.uni-hannover.de \\
\hline vifamath & $\begin{array}{l}\text { "The Virtual Library of Mathematics" - a meta } \\
\text { online catalogue }\end{array}$ & vifamath.de \\
\hline myLibrary & a physical library known by the subject & - \\
\hline $\operatorname{arXiv}$ & $\begin{array}{l}\text { "Open e-print archive with over [...] } 10000 \text { [arti- } \\
\text { cles] in mathematics" }\end{array}$ & arxiv.org \\
\hline ResearchGate & "a network dedicated to science and research" & researchgate.net \\
\hline mathoverflow & $\begin{array}{l}\text { "a question and answer site for professional math- } \\
\text { ematicians" }\end{array}$ & mathoverflow.net \\
\hline myColleagues & personal colleagues as math search interface & - \\
\hline MSC-Map & $\begin{array}{l}\text { "accessing math via interactive maps" based on an } \\
\text { MSC metric }\end{array}$ & map.mathweb.org \\
\hline arXiv-Catchup & $\begin{array}{l}\text { an interface for catching up with the newest arti- } \\
\text { cles in math }\end{array}$ & arxiv.org/catchup \\
\hline FormulaSearch & $\begin{array}{l}\text { "allows to search for mathematical formulae in } \\
\text { documents indexed in zbMath" }\end{array}$ & zbmath.org/formulae \\
\hline Bibliography & a bibliography as math search interface & \\
\hline
\end{tabular}

Table 1. The RGI Elements in the Study

\subsection{The RGI Elements}

As we want to cover a broad range of different types of math search interfaces we opted for a set of $17 \mathrm{mSIs}$ as RGI elements - ranging from standard mSIs like 
"Zentralblatt Mathematik (zbMath)" or "MathSciNet" via social media platforms like "mathoverflow" to scientific prototypes like the "MSC map" interface (MSC = Math Subject Classification, see [17]). To avoid being limited to digital mSIs, we included traditional search situations like asking colleagues or personal office spaces as well. Table 1 summarizes the 17 elements used in the RGIs and gives short descriptions - the ones from their websites where available - and their web addresses if applicable. Note that wikis (e.g., "Wikipedia" or "PlanetMath") were excluded as the tension between searching for articles versus encyclopedia entries was perceived problematic in the pilot study, so we opted for the former. As we were only interested in the search behavior of mathematicians we disregarded mathematical software whose main task is computation or verification.

\subsection{The RGI Set-Up}

At the beginning of each interview the interviewer introduced the interviewee to all mSIs based on print-outs. Both the home page with its search facilities and the search result pages were discussed. The front page of each print-out presented the homepage initialized with the phrase "Cauchy sequence" in the search box if applicable. The back page displayed the search result wrt to this query. For mSIs with special features extra pages were attached. For FormulaSearch the $\mathrm{LT}_{\mathrm{E}} \mathrm{X}$ query corresponding to $? a_{? n} \in \mathrm{N}$ was used.

An RGI interview iterates the following process until the interviewee's individual construct space seems to be exhausted:

$i$. The interviewee randomly chooses three RGI elements.

ii. He declares which two of the three elements seem more similar.

iii. He determines the aspect under which these two are more similar and the aspect under which the third one is different. Those aspects are the "poles" of an interviewee-dependent evaluation dimension, the so-called "construct". To get a sense of what the users consider important properties of mSIs, we extended this set-up by encouraging most interviewees to judge the "fitness" of each mSI for mathematical search. As is typical with RGIs, the interviews were very intense. Therefore, the findings are not only based on the actual data elicited in the RGI but also on the deep discussions taking place during each interview.

\subsection{The RGI Data}

We conducted interviews with 24 people, all of which were interested in accessing math on the web. Out of these, 18 had a degree in mathematics. For the final analysis we decided to use 22 RGIs: interviews with a group of 11 professional mathematicians working in a scientific environment ("INMATH"), a group of 5 content experts for mathematical information ("INFOMATH"), and a group of 6 non-mathematicians ("NOMATH"). Only 3 of the participants were female.

Each interview took between 1.75 and 3 hours, in which an average of 4 constructs were elicited. The INMATH group created 50 constructs, INFOMATH reported 28 constructs and NOMATH 29 constructs. The rating scale for these 107 elicited constructs was a 7-point Likert scale. 


\section{Findings}

As already mentioned, the RGI method is semi-empirical. This means that there will be a quantitative and a qualitative analysis of the data gathered. Due to space limitations we will focus on presenting and interpreting the most interesting, statistically significant quantitative results in form of dendrograms and qualitative results in form of patterns. Note that here, the theory emerges from the data, thus, it provides us with patterns but not with proofs.

With the Generalized Procrustes Analysis (GPA) method (see [2]) 3dimensional data matrices can be analyzed with a multivariate statistical technique. In particular, in our RGI we can compare the individual (dim 1) natural language constructs $(\operatorname{dim} 2)$ rated on our fixed set of mSIs $(\operatorname{dim} 3)$. We conducted a GPA with Idiogrid for each data set and refer to 11 for a detailed description of an analoguous GPA procedure. To provide a shared set of (virtual) standard constructs on which the individual ratings of the RGI elements of each interviewee can be compared, the GPA method produces "abstract constructs" of the form "Con_i - ConOpo_i" with poles "Con_i" and "ConOpo_i".

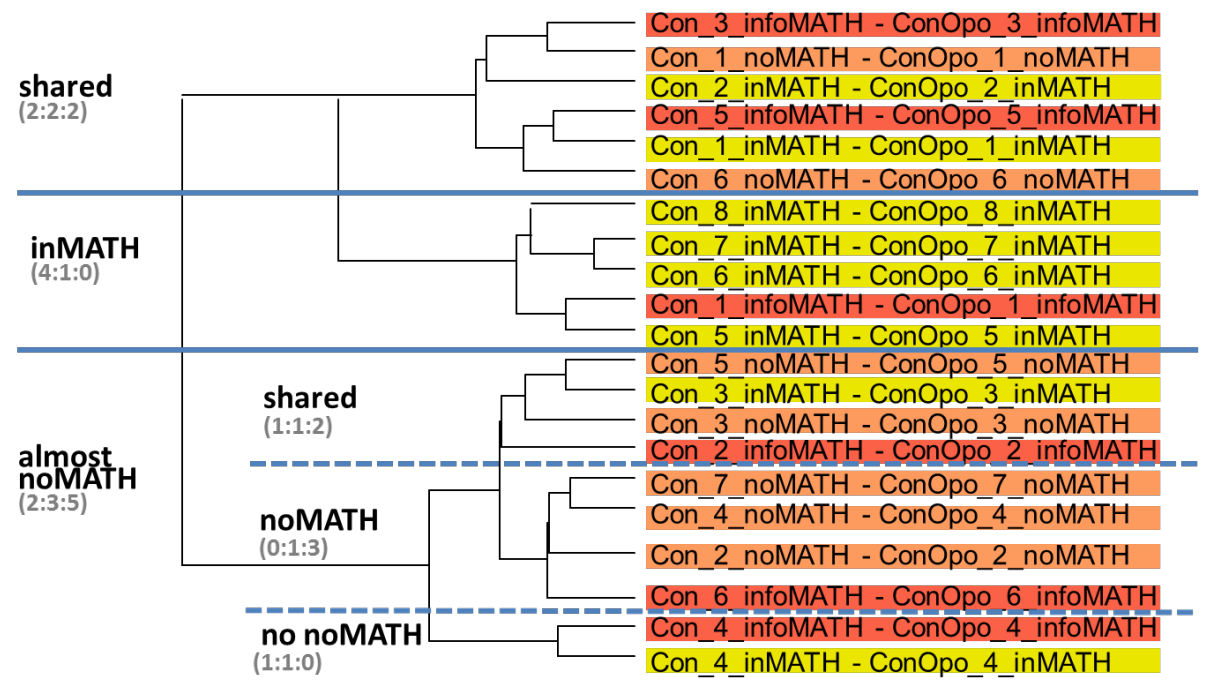

Fig. 1. Dendogram of the Abstract Construct Clusters (wrt. Euclidean distance and Ward clustering) of INMATH, INFOMATH and NOMATH: we can clearly discern a "common" cluster, which is equally shared by all three, a strong INMATH cluster and a fairly strong NOMATH cluster.

Based on a pre-study we suspected a distinction of the interviewee group not only into mathematicians and non-mathematicians, but into research mathematicians, mathematics practitioners and non-mathematicians. Therefore we compared the element evaluations of the INMATH, INFOMATH, and NOMATH group. We subjected the union of the group-specific sets of abstract constructs to a cluster analysis run by OpenRepGrid resulting in the dendrogram in Fig. 1 . Recall that dendrograms are a visual representation of correlation data. Two constructs in Fig. 1 are closely correlated, if their scores on the RGI elements 
are similar. The distance to the next upper level of two constructs/groups of constructs indicates this relative closeness. Please note that we left out the scale in the dendrograms, as we are not interested in the absolute numbers, only in their relative groupings. This also means, that we won't use arguments in our discussion of findings based on this scale. Nevertheless, we can for example, conclude from Fig. 11 that Con_6_inMATH and Con_7_inMATH are the most correlated constructs. For the conversion of Idiogrid data to OpenRepGrid data we developed the according software.

The interview data seen in Fig. 1 indeed suggest a difference between how people in the INMATH, INFOMATH and the NOMATH group think about mSIs. The INFOMATH interviewees' point of view lies between the one of INMATH and NOMATH subjects. In particular, there are INFOMATH abstract constructs in every cluster and there is no cluster dominated by the INFOMATH abstract constructs. As this user group dilutes possible similarities or dissimilarities wrt the user group in focus - the professional mathematicians - we further on only analyzed the INMATH and NoMATH data in depth. From here on we will call INMATH members "mathematicians" and NOMATH members "nonmathematicians".
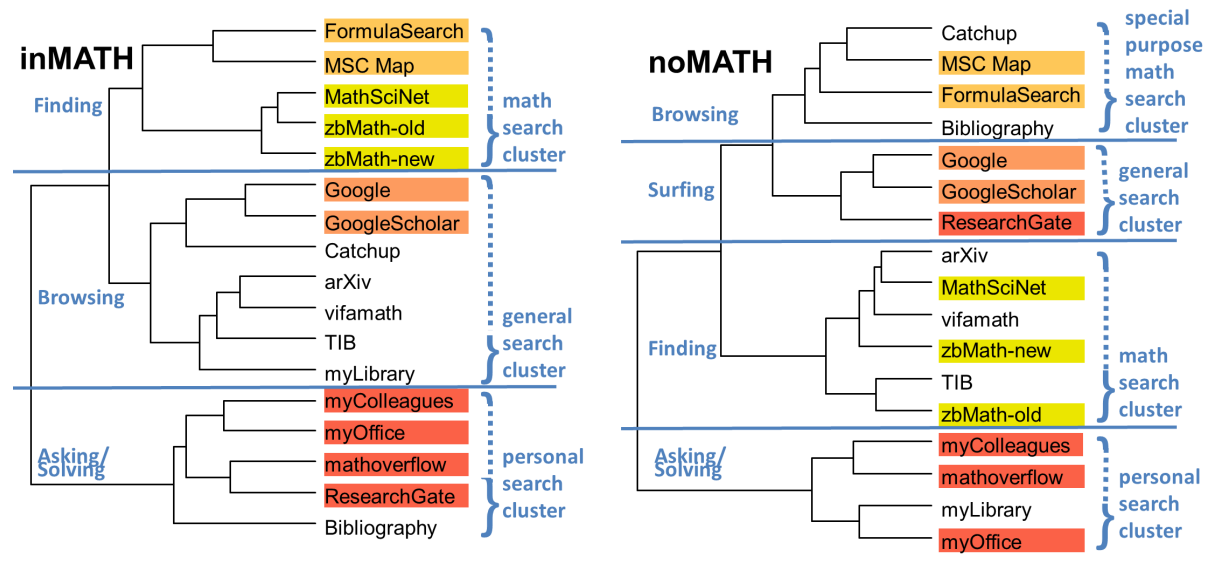

Fig. 2. Cluster Dendrograms of mSI Elements for INMATH and NOMATH

Fig. 2 gives a visualization of the element clusters of group INMATH resp. NOMATH as dendrograms. The difference between the clusters is evident; we will elaborate the interpretations in the next paragraphs.

There are three main element clusters for INMATH in Fig. 2. Clearly, one of these contains the mSI elements whose main purpose it is to find mathematical content ("math search cluster"). In the math search cluster both, FormulaSearch and MSC-Map, are innovative mathematical services, nevertheless they are identified as being most similar to the standard mSIs zbMathNew, MathSciNet and zbMath0ld. This shows that

Pattern 1: "Mathematicians do not assess mSIs based on familiarity." 
Another cluster includes all $\mathrm{mSI}$ elements that provide a personal touch in the search process ("personal search cluster"). Here, the term "personal" labels the interactive adaptation and customization of the search or search results in a process driven by human interactions. In the interviews it became quite clear, that anything involving human beings or communities was highly distinctive and predominantly highly appreciated. So

Pattern 2: "Mathematicians trust human and community resources."

Note that we don't mean a naive trust here, but a trust given the sensible precautions. Even though the element clusters of the NOMATH interviewees also include a personal search cluster (see Fig. 2), the elements Bibliography and ResearchGate are missing and replaced by myLibrary. The INMATH participants explicitly commented that they don't have confidence in the librarians' expertise in math. Interestingly, mathematicians showed a lot of skepticism wrt ResearchGate but not because they could not rely on the links the ResearchGate members would provide, but rather because they mistrusted ResearchGate's competence in judging the relevance of links. An indication of this is also given by the well-known observation that mathematicians like anecdotes about fellow mathematicians like no other community of practice.

The third cluster groups the remaining elements. Noticeably Google and Google-Scholar, which mathematicians nowadays use heavily for mathematical searches, are in this cluster. Nevertheless, these elements are not specific to math search, therefore we label this cluster as the "general search cluster".

According to ZHAO's usability study in [27, mathematicians use "three main approaches: general keyword search, browsing math-specific resources and personal contact." This can also be seen in our three clusters for the INMATH group.

For the NOMATH element clusters we only want to point out that the clusters are indeed very different from the ones in the INMATH dendrogram. For example, for mathematicians the mSIs MathSciNet, zbMathNew and zbMathold correlate the highest, whereas for non-mathematicians each of them correlates more with a different element than with each other. The only similarities seem to be the obvious correlation between Google and Google-Scholar, and the same very high correlation distance between the personal search cluster and the others.

For a more precise qualitative analysis consider the dendrogram in Fig. 3 . First we decided on fitting categories/subcategories for each cluster. We looked, for instance, at the first main cluster and decided on the category "fit for math". Then we elaborated on its four subclusters, e.g., for the fourth cluster we selected "preconditions for search" as a subcategory. Note that there are blue-colored abstract constructs "Con_i - ConOpo_i" among the constructs. We can interpret them now as characteristic constructs of the corresponding major subcluster, so we associate each abstract construct with its subcategory. Out of convenience, we call them by their explicit pole name together with the corresponding data set, thus we say for example, "Con_4_inMATH 'means' math specificity".

According to Kuhltau ET AL. in 13, 14 the information search process can be described by a six-phase framework consisting of initiation (prompting a 


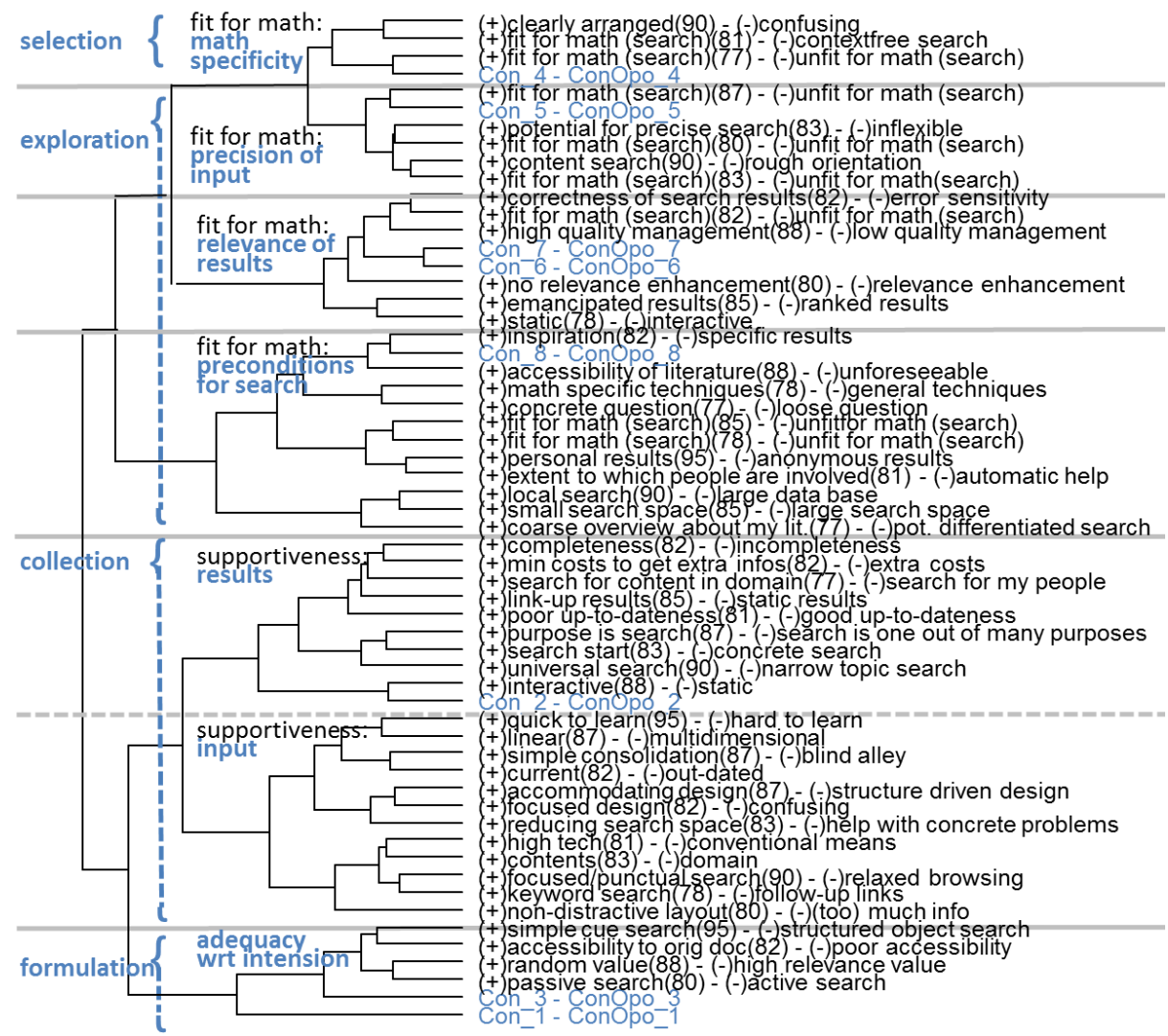

Fig. 3. Cluster Dendrogram of Construct Clusters in INMATH. The first two levels of the dendrogram were contracted for a more readable image. Moreover, the numbers in parentheses in each construct encode the individual interviewee issuing it.

search), selection (identifying information needs), exploration (pondering available tools and thus search strategies), formulation (formalizing search queries), collection (gathering information and goal-oriented cherry picking in search results), and search closure (giving up on the search). In our study we are not interested in the entire search process, but in the interactions with the user interface, so we focus on the iterative acts of selection, exploration, formulation and collection. In these phases a user seeking information translates a search intension into a query or series of queries optimizing for the relevance of the final collection of search results. Interestingly, the four phases are mirrored in the construct clusters of the INMATH group (see Fig. 3 on the left).

To obtain a ranking for the abstract constructs consider the structure coefficients of the abstract constructs (wrt their ratings on the three main principal components $P C_{i}$ ) for each interviewee group in Fig. 4 and 5 The Euclidean length of the resp. 3-dimensional construct vector indicates its construct's relevance. To distinguish between two abstract constructs that are in the same subcluster in Fig. 3. we compare their structure coefficients. For any distinctive 


$\begin{array}{llrrrl}\text { Meaning } & & \text { PC_1 } & \text { PC_2 } & \text { PC_3 } & \text { Length } \\ \text { relev. of results: driven by user } & \text { Con_6_inMATH } & -0.61 & 0.52 & 0.52 & 0.96 \\ \text { relev. of results: driven by data } & \text { Con_7_inMATH } & -0.72 & 0.09 & 0.63 & 0.96 \\ \text { precision of input } & \text { Con_5_inMATH } & -0.59 & -0.61 & -0.37 & 0.93 \\ \text { adequacy: search philosophy } & \text { Con_1_inMATH } & 0.03 & 0.70 & -0.59 & 0.92 \\ \text { math specificity } & \text { Con_4_inMATH } & -0.81 & -0.20 & -0.35 & 0.90 \\ \text { adequacy: trustfulness } & \text { Con_3_inMATH } & 0.05 & 0.72 & -0.03 & 0.72 \\ \text { supportiveness: result } & \text { Con_2_inMATH } & -0.36 & 0.50 & -0.34 & 0.70 \\ \text { preconditions for search } & \text { Con_8_inMATH } & 0.34 & -0.05 & 0.49 & 0.60\end{array}$

Fig. 4. Abstract Construct Ranking in INMATH via Structure Coefficients

deviation we take a closer look in the biplots for the resp. PC-dimension and elaborate on its meaning.

It is obvious that the rankings of the INMATH group are distinct from the ones of the NOMATH group. What mathematicians care about the most is the relevance of the search result with respect to their search intension. So they seek interfaces and databases that allow them to formulate precisely that in accordance with the respective search philosophy they want to apply (Con_6_inMATh, Con_7_inMath, Con_5_inMath, Con_1_inMath). As this describes a search process that enables the user to find exactly what he is looking for, we have

Pattern 3: "Finding is the primary mathematical search task."

Note that the math search cluster of the INMATH group in Fig. 2 also has a clear focus on "finding". For the NOMATH math search cluster this is much less clear, e.g., the vifamath mSI, which concentrates on collecting mathematical information (from legacy math articles to images of mathematicians), but not on its findability, thus mimicking a physical math library without noticeable presence of other people. The interviewer observed that interviewees aligned the distinct kinds of search like finding, browsing or solving with the clusters, but that the evaluation of search activities was different for mathematicians and non-mathematicians. The former had a clear preference for finding, followed by browsing and solving/asking, and even a hint of rejection for surfing. In contrast, the NOMATH participants indicated a preference for browsing and surfing, followed by solving/asking and finally finding. Note that the position of "finding" in this ranking may be well due to the fact, that only one participant in the NOMATH group worked in a scientific environment.

It is conspicuous that even though there was an obvious mSI cluster with respect to "people" (the personal search cluster) for the INMATH group in Fig. 3 . there is no appreciation of "socialness" in their ranked list of constructs in Fig. 4 . In particular, mathematicians distinguish certain mSIs, i.e., the tools, as socially driven, but as professionals they do not appreciate "socialness" as a value per se in their evaluation schemes. In the theory of "Communities of Practice (CoP)" 15, practices are not only typical customs shared within a community, but they are tools that define the community. Whereas in other CoPs social interaction is a tool for achieving social bindings, in the mathematical CoP, social interaction is a tool for doing mathematics, i.e., it is a mathematical practice. Therefore, we note that 
Pattern 4: "Mathematicians appreciate social interaction as a mathematical tool. In particular, it is a mathematical practice to collaborate and exchange feedback."

In this sense, we confirm BRown's dictum in 11 that mathematicians may rely more heavily on their social network than other disciplines.

$\begin{array}{llcccl}\text { Meaning } & & \text { PC_1 } & \text { PC_2 } & \text { PC_3 } & \text { Length } \\ \text { input design } & \text { Con_1_noMATH } & 0.11 & 0.61 & 0.71 & 0.94 \\ \text { math specificity } & \text { Con_5_noMATH } & -0.90 & 0.21 & 0.15 & 0.94 \\ \text { flex.: what and when } & \text { Con_2_noMATH } & -0.92 & -0.01 & 0.07 & 0.92 \\ \text { adequacy } & \text { Con_3_noMATH } & -0.54 & -0.70 & -0.15 & 0.90 \\ \text { flex.: what } & \text { Con_4_noMATH } & -0.10 & -0.42 & 0.76 & 0.87 \\ \text { flex.: where and how } & \text { Con_7_noMATH } & -0.80 & 0.19 & 0.01 & 0.82 \\ \text { usability and interactivity } & \text { Con_6_noMATH } & 0.28 & -0.56 & 0.43 & 0.76\end{array}$

Fig. 5. Abstract Construct Ranking in NOMATH via Structure Coefficients

Let us recall from Fig. 1, that some mSI elements were in a subcluster shared by all three user groups. That is, with respect to these constructs the INMATH, INFOMATH as well as NOMATH interviewees agreed on the evaluation of the given mSIs. In particular, mSI scores correlate on Con_3_infoMATH ("usability"), Con_1_nomath ("input design"), Con_5_infomath ("simple design"), Con_6_nomath ("usability and interactivity"), and Con_2_inMATH ("supportiveness:result"), Con_1_I NMATH ("adequacy: search philosophy").

We note the different flavor of the non-INMATH constructs versus the INMATH constructs. Where the former aim for design aspects, the latter are only concerned with fitness of the mSI for achieving the search intension. It becomes even clearer if we consider the phrasing "usability" in the non-INMATH group and "supportiveness" in the INMATH group: Whereas usability is a neutral measure for all kinds of qualities while using an object, supportiveness is a taskoriented requirement in the use-flow of a human person. The media-theoretic difference is that the first doesn't tell us anything about whether the user adopts a mSI as a mere tool or as a medium (in the sense of MCLUHAN as "any extension of the human body [...] as a side-effect of a technology" [18, p. 564], i.e., a technology that empowers its users):

Pattern 5: "Mathematicians aim at adopting a search tool as a medium."

One consequence is that once they have adopted it as a medium, they won't easily change to other media. Not surprisingly, this shared construct cluster also supports a long-standing belief that

Pattern 6: "Mathematicians appreciate function over form."

Even though the mSI elements' scores were highly correlated in the shared cluster, their respective conceptualization can still disagree. To understand the conceptualization, we look at the meanings of the distinct constructs and the location of an element wrt constructs. For instance, for mathematicians Google-Scholar enables a top-down approach (as search philosophy =Con_1_I NMATH) 
by using a very general technique of ranking the search results (high supportiveness for presenting search results =Con_2_I NMATH), but offers a very textual input design (=Con_1_NoMATH) and a medium-rated effectiveness (as part of usability = Con_6_NoMATH) for non-mathematicians. Here, note that the evaluation by the mathematicians concerns the outcome, whereas the non-mathematicians rather assess it by the input. This argument can be made more generally, as the resp. construct clusters for the INMATH resp. NOMATH groups favor the result resp. the input. Interestingly one cluster category of the INMATH group didn't make it into the consensus grid. In particular, the category "supportiveness of input" has no representative among the abstract constructs of InMATH. We conclude

Pattern 7: "Mathematicians care more for the outcome than the input."

This also means that mathematicians seem to be willing to trade input hardships (like more complex interfaces) for output satisfaction (i.e., having perfect precision - all found results fit the search intension- and recall - all fitting results were found).

In Fig. 1 we observe that the constructs Con_5_inMATH, Con_6_inMaTH, Con_7_INMATH, and Con_8_INMATH are part of an abstract construct cluster only containing INMATH constructs; they are the enhanced (yellow colored) constructs in Fig. 4. Here, the math interfaces scored similarly according to the attributes "relevance of results: driven by user", "relevance of results: driven by data", "precision of input", and "preconditions of search". Thus, we can interpret that a mathematical search interface that empowers the user by enabling him to finetune the search query is considered to strongly improve the relevance of the result. This interpretation is supported by Pattern 7, thus we note that

Pattern 8: "Mathematicians want to be empowered in the search process."

Moreover, mathematicians obviously realize that this precision comes at a cost: the underlying data have to be structured enough. Therefore, if the data do not allow such a fine-tuning right away, they are willing to iteratively refine their query themselves. A direct consequence seems to be that mathematicians want as much support in formulating a search query as they can get. Whereas non-mathematicians will agree that Pattern 7 is different from their own approach, wrt the above consequence their attitude might be different as the pattern describes the disregard of input facilities by mathematicians and the latter the total investment of time and energy towards satisfying the search intension. We already observed that this is a cluster of elements marked by mathematicians only. That is, this kind of evaluation scheme didn't occur to non-mathematicians, thus it isn't a dominant one.

For Pattern 3 we argued with the abstract construct ranking within Fig. 4. Interestingly, three of the four first ranked items in that list belong to the uniquely mathematical cluster. The fourth one (Con_8_inMATH, "preconditions for search") occurred in the INMATH group only, that is, it discriminates between mathematicians' and non-mathematicians' search behavior ever more. As mathematicians take the preconditions for search into account in the exploration phase of an 
information search process, they value their anticipation of the search outcome. This has two consequences:

Pattern 9: "Mathematicians base their information search process on transparency of the search result."

Additionally, if they put a lot of thought into the exploration phase, they expect to be rewarded by a good search result. So we hold

Pattern 10: "Mathematicians expect to find meaningful information in the search result."

In the interviews, it was striking how much awe Google evoked. Pattern 10 solves this riddle: Considering the low amount of work to be invested in the exploration phase, the expectations towards the search results are really low. Therefore, the relevance of Google searches amazes mathematicians tremendously.

\section{Understanding the Mathematical Perspective on mSIs: an Example}

To see the utility of the elicited patterns, we will now discuss the mSIs MathSciNet and zbMathNew under a mathematical perspective, which is informed by our elicited patterns.

Let us start with MathSciNet as seen in Fig. 6 and zbMathNew shown in Fig. 7. From above we know that mathematicians don't discern between MathSciNet and zbMathNew. This immediately raises the question why this might be the case. Evidently both layouts use a lot of vacuity to focus the users' attention and use bright colors sparingly. But we know because of Pattern 6 , that the form is not im-

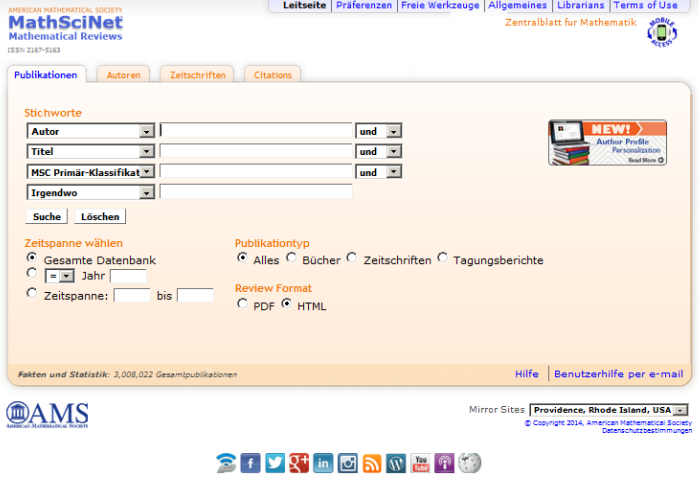

Fig. 6. mSI of MathSciNet portant to mathematicians, so the reason for their alignment cannot stem from these observations. Unfortunately, at first glance the similarity of the start page already ends here: zbMathNew provides a simple search, i.e., a one-step search, MathSciNet a multi-dimensional, structured search. Moreover, zbMathNew offers innovative extra services like mathematical software search and formula search, MathSciNet an extra citation service. The former offers inline search fields to specify the search. The latter provides social media connections. If we look at the search result page of each, we will find that there are as many differences. 
Now let us take a closer look, for example, at the difference between zbMathNew's simple search and MathSciNet's structured search. We know because of Pattern 7 , that mathematicians value the outcome higher than the input. Therefore, as zbMathNew offers not only the functionality of MathSciNets multi-dimensional search via inline search fields in the simple search but also choicewise a link to a structured search, the functionality seems to be the same for mathematicians. The input inefficiencies can be neglected, the potential outcome is the same.

What about the clear differences in functionality in these mSIs? Note that the social media links weren't recognized once in the interviews with the mathematicians, which also fits Pattern 4 , stating that they appreciate the communities' practices, but not the links to a community themselves. Then,

Edited by.

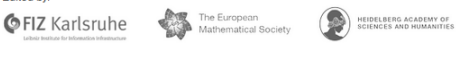

Fig. 7. mSI of zbMathNew it seems rather evident that $\mathbf{z b}-$ MathNew offers more functionality, as MathSciNet's extra functionality consists only of the citations index. So shouldn't Pattern 8 kick in and lead to a distinctive perception of both systems?

We can counter-argue with two patterns. On the one hand, Pattern 3 tells us that finding is the major kind of search a mathematician is conducting. The additional services zbMathNew provides the user with are essentially no services that support finding, they rather support browsing. This is clear for the mathematical software search. The facetted search with its abilities to refine a search in the process also supports browsing behavior explicitly. In contrast, the formula search feature was designed for finding, but in the interviews, mathematicians indicated that they simply don't believe in the finding capability of the software (unfair as it is). In [27], interestingly, a similar phenomenon was observed. The underlying reason for this disbelief could lie in Pattern 5, namely that they have adopted zbMathNew as a medium, and that uses string search. Therefore, their conceptualization of this service doesn't fit yet and is a challenge to change.

On the other hand, zbMathNew is rather new. The older version didn't have as many relevant extra features as this new one. Thus, Pattern 5 strikes again. Quite a few interviewees reported that they use MathSciNet and even when they became aware that zbMathNew has more to offer now, they didn't mention any intention to change over.

We can summarize that the patterns help us understand the perception of mathematicians much better. This new-found understanding in turn triggers new design challenges and ultimately better, more math-oriented designs. 


\section{Conclusion}

We have presented an RGI study that was concerned with mathematical search interfaces. To be able to understand the idiosyncracies of mathematicians, we interviewed mathematicians as well as non-mathematicians, with a focus on the former. From the quantitative data and its qualitative interpretation several patterns emerged:

P 1 "Mathematicians do not assess mSIs based on familiarity."

P 2 "Mathematicians trust human and community resources."

P 3 "Finding is the primary mathematical search task."

P 4 "Mathematicians appreciate social interaction as a mathematical tool. In particular, it is a mathematical practice to collaborate and exchange feedback."

P 5 "Mathematicians aim at adopting a search tool as a medium."

P 6 "Mathematicians appreciate function over form."

P 7 "Mathematicians care more for the outcome than the input."

P 8 "Mathematicians want to be empowered in the search process."

P 9 "Mathematicians base their information search process on transparency of the search result."

P 10 "Mathematicians expect to find meaningful information in the search result."

With these patterns many design issues for mSIs can be understood and elaborated on much deeper now.

For instance, LiBBRECHT posed in 16 the question whether (mathematical) search queries may become too precise (so that the search result becomes too small). But this question does only make sense for browsing queries not for finding queries. The Pattern 8 suggests that the solutions should be finetuned to the distinct kind of searches. If that is not possible, the default case should be "finding" because of Pattern 3 .

Pattern 5 indicates that a change from one tool to another is not easily done by mathematicians. In particular, a change of media will only occur if the innovation is disruptive, a mere incremental innovation won't suffice. Therefore, phrasing a major change (like the one from zbMathold to zbMathNew) as a mere update won't convince mathematicians to switch, and because of Pattern 7, neither will an announcement of change that essentially points to the new Google-like layout of the homepage.

Moreover, our data suggest that the search approach "finding" is used by mathematicians predominantly when interacting with elements from the math search cluster, "browsing" when interacting with mSIs in the general search cluster and "solving/asking" when using elements in the personal search cluster. Thus, we can look for the properties of the resp. cluster to extend mSIs by more search approaches. Note that Google is best-known for its browsing qualities, only for specific kinds of queries it is now also successful in finding. Under this aspect Google is also often used by mathematicians.

Our future work is concerned with general design implications based on the foundational work conducted in this paper. For example, one simple consequence concerns the development process of math user interface development: Specify 
the user group of your math service beforehand and appreciate the credo of "participatory design" that strongly admonishes developers to acknowledge the fact that "You are not the user!". In our study, e.g., the mathematics practitioners turned out to be different from the professional mathematicians. Another consequence might be that we should make mathematicians more effective by supporting their interventions in formulating a search query.

Finally, we like to note that the RGI methodology - even though strenuous at times - seems to be a worthy methodology for use with mathematicians.

Acknowledgement I thank all my interviewees for their motivation and patience with the RGI method. Moreover, I appreciated the supportive work environment at zbMath, especially discussions with Wolfram Sperber. This work has been funded by the Leibniz association under grant SAW-2012-FIZ. The final publication is available at http://link.springer.com.

\section{References}

[1] Cecelia M. Brown. "Information Seeking Behavior of Scientists in the Electronic Information Age: Astronomers, Chemists, Mathematicians, and Physicists." In: JASIS 50.10 (1999), pp. 929-943.

[2] J. Gower. "Generalized procrustes analysis". In: Psychometrika 40 (1 1975), pp. 33-51. ISSN: 0033-3123.

[3] James W. Grice. "Idiogrid: Software for the management and analysis of repertory grids". In: Behavior Research Methods, Instruments, 83 Computers 34 (2002), pp. $338-341$.

[4] Marc Hassenzahl and Rainer Wessler. "Capturing Design Space From a User Perspective: The Repertory Grid Technique Revisited". In: International Journal of Human-Computer Interaction. 3rd ser. 12 (2000), pp. 441-459. ISSN: 1044-7318.

[5] Stephanie Heidecker and Marc Hassenzahl. "Eine gruppenspezifische Repertory Grid Analyse der wahrgenommenen Attraktivität von Universitätswebsites". In: Mensch $\&$ Computer. Ed. by Tom Gross. Oldenbourg Verlag, 2007, pp. 129-138.

[6] Morten Hertzum and Torkil Clemmensen. "How do usability professionals construe usability?" In: Int. J. Hum.-Comput. Stud. 70.1 (2012), pp. 2642.

[7] Devi Jankowicz. The Easy Guide to Repertory Grids. Wiley, 2003. ISBN: 0470854049 .

[8] Melanie Kellar, Carolyn R. Watters, and Michael A. Shepherd. "A field study characterizing Web-based information-seeking tasks". In: JASIST 58.7 (2007), pp. 999-1018.

[9] George Kelly. "International Handbook of Personal Construct Technology". In: John Wiley \& Sons, 2003. Chap. A Brief Introduction to Personal Construct Theory, pp. 3-20. 
[10] Tim Kitchen. The European Digital Mathematics Library: Usability Study. URL: https://wiki . eudml.eu/eudml-w/images/D6.1.pdf (visited on 03/14/2014).

[11] Andrea Kohlhase. Framings of Information: Readers' Perception of Information Sources in Spreadsheets. Tech. rep. 30. Jacobs University, Mar. 2013. URL: kwarc.info/ako/pubs/tr_hsi_2013.pdf.

[12] Andrea Kohlhase. "Human-Spreadsheet Interaction". In: Human-Computer Interaction - INTERACT 2013. Ed. by Paula Kotzé et al. Vol. 8120. LNCS. Heidelberg: Springer, 2013, pp. 571-578. ISBN: 978-3-642-40497-9.

[13] Carol C. Kuhlthau, Jannica Heinström, and Ross J. Todd. "The information search process revisited: is the model still useful?" In: ir information research 13.4 (2008).

[14] Carol Collier Kuhlthau. Seeking meaning. A process approach to library and information services. 2. Libraries Unlimited, 2004, pp. XVII, 247.

[15] Jean Lave and Etienne Wenger. Situated Learning: Legitimate Peripheral Participation (Learning in Doing: Social, Cognitive and Computational Perspectives S.) Cambridge University Press, 1991.

[16] Paul Libbrecht. "Escaping the Trap of Too Precise Topic Queries". In: MKM/Calculemus/DML. (Bath, UK, July 8-12, 2013). Ed. by Christoph Lange et al. CEUR Workshop Proceedings 1010. Aachen, 2013, pp. 296309. URL: http://ceur-ws.org/Vol-1010.

[17] Mathematics Subject Classification (MSC) SKOS. 2012. URL: http:// msc2010.org/resources/MSC/2010/info/ (visited on 08/31/2012).

[18] Marshall McLuhan. Understanding Media: The Extensions of Man (Critical Edition), edited by W. Terrence Gordon. Corte Madera. CA: Gingko Press, [1964] 2003.

[19] P. Morville and J. Callender. Search Patterns: Design for Discovery. O'Reilly Media, 2010. ISBN: 9781449383060.

[20] R. Navarro-Prieto, M. Scaife, and Y. Rogers. "Cognitive strategies in web searching". In: Proceedings of the 5th Conference on Human Factors 83 the Web. 1999.

[21] openrepgrid.org. URL: http://www .openrepgrid.org (visited on 03/14/2014).

[22] Stuart J. Russell and Peter Norvig. Artificial Intelligence - A Modern Approach. Upper Saddle River, NJ: Prentice Hall, 1995.

[23] Ben Shneiderman, Don Byrd, and W. B Croft. "Clarifying Search: A UserInterface Framework for Text Searches". In: (1997). ISSN: 1082-9873.

[24] Felix B. Tan and M. Gordon Hunter. "The Repertory Grid Technique: A Method for the Study of Cognition in Information Systems". English. In: MIS Quarterly 26.1 (2002), pp. 39-57. ISSN: 02767783.

[25] Robert S. Taylor. "The process of Asking Questions". In: American Documentation 13.4 (1962), pp. 391-396. DOI: 10.1002/asi.5090130405.

[26] Kevin Wise, Hyo Jung Kim, and Jeesum Kim. "The effect of searching versus surfing on cognitive and emotional responses to online news". In: Journal of Media Psychology: Theories, Methods, and Applications 21.2 (2009), pp. 49-59. DOI: 10.1027/1864-1105.21.2.49.

[27] Jin Zhao, Min-Yen Kan, and Yin Leng Theng. "Math Information Retrieval: User Requirements and Prototype Implementation". In: Proceed- 
ings of the 8th ACM/IEEE-CS Joint Conference on Digital Libraries. JCDL '08. Pittsburgh PA, PA, USA: ACM, 2008, pp. 187-196. ISBN: 9781-59593-998-2. 